\title{
Effect of lateralized gaps in noise on the cutaneous blink reflex in humans
}

\author{
GEOFFREY HAMMOND \\ University of Western Australia, Nedlands, Australia
}

\begin{abstract}
The early (R1) and late (R2) components of the cutaneous blink reflex in right-handed humans were recorded in three experiments to examine the lateral symmetry of a simple excitatory process in their brainstem pathways and of the control of the excitability of the pathways by gaps in acoustic noise. Experiment 1 showed that a stimulus below R1-elicitation threshold increased the excitability of the right R1 pathway more than the left but that the rate of decay was similar on both sides. Experiment 2 showed that a brief unilateral gap in noise affected the R1 and R2 reflex pathways bilaterally. Experiment 3 showed that R2 varied with gap duration and that gaps to the left and right ears had indistinguishable effects. The finer temporal resolution of events in the right sensory field of right-handers seen in psychophysical judgments is not seen in the descending control of brainstem excitability.
\end{abstract}

Innocuous stimuli that appear just before reflex elicitation affect reflex size, with inhibition of reflex amplitude the most common outcome. Reflex modification is a robust phenomenon both in its size and its presence in a range of reflexes, species, and stimulus configurations (see reviews by Hoffman \& Ison, 1980; Ison \& Hoffman, 1983). The robustness of reflex modification and its automatic nature make it a useful means of evaluating sensory processing of the leading reflex-modifying stimulus (Hoffman \& Ison, 1992). The sensitivity of reflex size to the properties of the reflex-modifying stimulus should also make reflex modification a useful tool to explore elementary processing asymmetries in nervous system function that might underlie higher level asymmetries of psychological function. Literature reviews have found that judgments of stimuli falling within the right sensory fields of right-handed humans are made with greater temporal resolution than those falling within the left sensory fields (Hammond, 1982; Nicholls, 1996). This result, which has been found with visual, auditory, and somatosensory stimuli, has been interpreted as revealing a greater temporal acuity of the left than of the right hemisphere in right-handers, which has in turn been proposed as a primitive mechanism of hemispheric specialization (Hammond, 1982). In particular, there has been a great deal of interest in the view that finer temporal resolution of events within the left than within the right hemisphere give it a greater ability to analyze events that change rapidly in time, and hence make it dominant for linguistic processing (see, e.g., Tallal, Galaburda, Llinas, \& von Euler, 1993).

The experiments reported here were supported by a grant from the Australian Research Council. Correspondence should be addressed to G. Hammond, Department of Psychology, University of Western Australia, Nedlands, WA 6907, Australia (e-mail: geoff@ psy.uwa.edu.au).
The threshold for detecting a brief interruption of otherwise continuous acoustic noise (a gap) is a widely accepted measure of temporal acuity (see, e.g., Eddins \& Green, 1995; Trehub, Schneider, \& Henderson, 1995). Ison and Pinckney (1983) showed that the acoustic blink reflex in humans was inhibited by gaps in noise that appeared at an appropriate interval before the blink-eliciting stimulus (ES), and that the minimum gap duration required for blink inhibition closely approximated estimates of gapduration thresholds from the method of limits in the same participants. Subsequent work by Ison, O'Connor, Bowen, and Bocirnea (1991) showed that inhibition of the rat's acoustic startle reaction by gaps in noise was lost following functional decortication induced by topical application of potassium chloride. This general finding has been extended recently by Kelly, Rooney, and Phillips (1996), who showed that gap-detection thresholds in ferrets (determined by a behavioral choice technique) were doubled following bilateral aspiration of the primary auditory cortex. Together, these experiments suggest that detection of gaps in noise by mammals requires the participation of the auditory cortex. Lateralized measures of gap detection might therefore reveal hemispheric asymmetries in temporal resolution of sensory information. The present experiments were done with two aims: first, to set the groundwork for using blink reflex modification to study brain asymmetries, and second, to determine whether this method would give converging evidence for finer temporal resolution of auditory processing in the left hemisphere of right-handers; the latter aim was addressed by assessing reflex modification as a function of the duration of gaps in noise presented separately to the left and right ears. The cutaneous blink reflex was used as the behavioral indicator because it is served by two partially separate brainstem pathways, a unilateral oligosynaptic pathway and a bilateral polysynaptic pathway; the first pathway results in a brief short-latency synchronized burst of electromyo- 
graphic (EMG) activity ipsilateral to the ES and the second in a prolonged longer latency burst of asynchronous EMG activity that appears bilaterally. The size of the first EMG component, R1, can be used to probe the excitability of a simple lateralized pathway in the brainstem, whereas the size of the second component, R2, can be used to probe the excitability of a complex interneuronal pool on both sides of the brainstem.

\section{EXPERIMENT 1}

Unambiguous interpretation of asymmetrical gapduration functions as cortical in origin requires that potential asymmetries in the time course of rapid neural processing in the brainstem can be ruled out or accounted for. When an ES of sufficient intensity to elicit an R1 is preceded by a similar stimulus below R1-elicitation threshold by a brief interval $(<12 \mathrm{msec})$, the R1 is augmented, showing that the leading stimulus evokes a transient increase in the excitability of the R1 pathway (Hammond $\&$ Plant, 1993). The time course of this transient excitability change offers one way of examining the time course of rapid processing on each side of the brainstem. In the first experiment, the time course of this increase in brainstem excitability changes in both left and right sides was examined by presenting pairs of stimuli to the left or right supraorbital nerve at stimulus onset asynchronies (SOAs) of $4-12 \mathrm{msec}$.

\section{Method}

Participants. Twelve volunteers ( 2 females) whose ages ranged from 17 to 24 years (median $=20$ years) participated. In this and the following experiments, all participants reported no hearing deficit and were self-reported right-handers who wrote exclusively with the right hand. All procedures had been approved by the University research ethics committee, and all participants gave informed written consent.

Apparatus and Procedure. The participants sat quietly with open eyes in a dimly lit sound-attenuating room while blink reflexes were elicited from their left and right eyes in separate trials. Blinks were elicited by a percutaneous stimulus presented alone (S2) or preceded by a leading percutaneous stimulus (S1). The participants were prepared with pairs of gold-plated stimulating electrodes over the left and right supraorbital nerves and recording electrodes over the left and right orbicularis oculi muscles. A ground electrode was attached to the mastoid process on one side. Threshold intensities required for brief $(0.2-\mathrm{msec})$ constant-current square-wave pulses to elicit an R1 on three successive occasions were determined separately for the left and right sides. EMG activity was amplified by conventional means (half-amplitude cutoff frequencies at 10 and $1000 \mathrm{~Hz}$ ), subjected to 12-bit analog-to-digital conversion at $4 \mathrm{kHz}$, and saved for later analysis. Five stimulus conditions were tested: S1-S2 pairs with SOAs of 4, 8, 10, and $12 \mathrm{msec}$, and S2 presented alone to establish control levels of R1 amplitude. The intensity of $\mathrm{S} 1$ was set at 0.8 times the $\mathrm{R} 1$ threshold, and that of $\mathrm{S} 2$ at $\mathrm{R} 1$ threshold. These intensities were above threshold for the lower threshold $\mathrm{R} 2$ component. The five stimulus conditions were presented randomly in blocks of 5 trials with an average intertrial interval of $10 \mathrm{sec}$ (range 6-14 sec). There were eight repetitions of each of the five stimulus conditions on each side for a total of 80 trials. The two sides were tested in an ABBA order; half of the participants received the stimuli in a LRRL sequence (with 20 trials in each trial block on each side) and the remainder received them in the reverse RLLR sequence.
Data analysis. Peak amplitudes (in $\mathrm{mV}$ ) of the $\mathrm{R} 1$ evoked by $\mathrm{S} 2$ and the area (in $\mathrm{mV} \cdot \mathrm{msec}$ ) of the R2 ipsilateral to the ES were scored off-line. The median R1 amplitude in each paired-stimulus condition on each side was expressed as a ratio of the median control amplitude obtained from the S2-alone trials on the same side. The ratios were log transformed before analysis, and geometric mean ratios (the arithmetic means of the transformed ratios) are reported with their standard errors $(S E)$.

\section{Results and Discussion}

Mean amplitude ratios at each SOA for R1 and the R2 ipsilateral to the ES delivered to the left and right sides are shown in Figure 1. S1 augmented the R1 elicited by S2, with all amplitude ratios greater than one; augmentation was most pronounced at the 4-msec SOA and decayed with increasing SOA, which accounted for a substantial proportion of the variance $\left(\eta^{2}=.60\right)$. These results replicate those reported previously (Hammond \& Plant, 1993) and indicate that $\mathrm{S} 1$, which was usually below threshold for reflex elicitation, caused a transient increase in the excitability of the R1 pathway. Second, there was an asymmetry such that $\mathrm{S} 1$ to the right supraorbital nerve augmented R1 more than an S1 of equivalent intensity (at 0.8 times elicitation threshold) to the left supraorbital nerve. This effect was present in 11 of the 12 participants and accounted for $14 \%$ of the variance $\left(\eta^{2}=.14\right)$. The more pronounced increase in the excitability of the R1 pathway in the right brainstem, although unexpected, might have some behavioral significance; Trevarthen (1996) has made a strong case for the primacy of asymmetrical motor

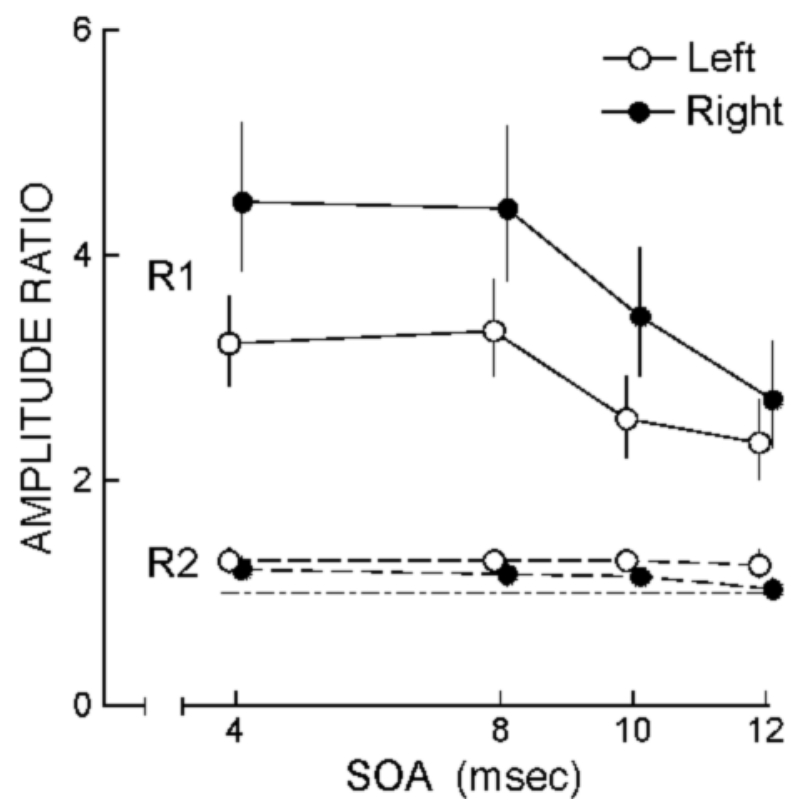

Figure 1. Mean amplitude ratios for $\mathrm{R} 1$ (the two upper plots) and the R2 ipsilateral to the ES (the two lower plots) for the left (open circles) and right (filled circles) sides as a function of stimulus onset asynchrony (SOA, in milliseconds) in Experiment 1. The error bars show $\pm 1 S E$, and the broken line shows an amplitude ratio of 1.0. The error bars for $R 2$ are smaller than the plotting symbol size in some conditions. The points are slightly displaced on the abscissa for clarity. 
systems in the brainstem, arguing that they are part of the foundation for the emergence of higher order cerebral asymmetries.

Despite this asymmetry in the amount of augmentation, the rate of decay was similar on each side. The influence of the different levels of augmentation on the left and right sides on the time course of decay was removed by expressing each participant's median R1 amplitude in the 8-, $10-$, and 12-msec SOA conditions as a ratio of his or her median R1 amplitude in the 4-msec SOA condition. The resulting mean ratios (with $S E$ s) for the three SOAs in order were $1.03(0.04), 0.79(0.07)$, and $0.73(0.07)$ for the left eye, and 0.98 (0.05), 0.77 (0.06), and $0.61(0.07)$ for the right eye. This result shows that the $\mathrm{R} 1$ pathways on each side of the brainstem respond to at least one form of imposed excitability change with a similar time constant. The result also matches an earlier report of a symmetrical time course of decay of augmentation of the monosynaptic spinal Hoffmann reflex with S1-S2 intervals of 2-17 msec (Hammond \& Mann, 1985). A report of asymmetrical recovery functions of the Hoffmann reflex, with more rapid recovery of excitability on the right-hand side in right-handers (Goode, Glenn, Manning, \& Middleton, 1980 ), probably reflects asymmetrical descending influences on the excitability of the spinal reflex pathway recruited during the long S1-S2 intervals (30-1,000 msec) used in that study rather than an asymmetry of the temporal characteristics of the spinal pathways themselves.

Because S1 (at 0.8 of R1 threshold) was suprathreshold for the lower threshold R2, this later component was present both ipsilateral and contralateral to the side of stimulation, whether evoked by the first member of an S1-S2 stimulus pair or the single stimulus (S2) of a control trial. Figure 1 shows that the R2 evoked on paired S1-S2 trials was augmented on both the left and right sides relative to the R2 evoked on the S2-alone control trials; this is consistent with a previous report (Hammond, Driscoll, \& Rowley, 1997). The amount of augmentation was much less than that seen with R1 and showed little decay with increasing SOA. This slow decay of augmentation relative to R1 may be a result of the longer duration of R2 than of R1: Even at the longest SOA on paired-stimulus trials (12 msec), S2 would have appeared while the R2 pathway was still active and so could have added to it. $R 2$ was augmented both ipsilateral and contralateral to the side of stimulation, with overall means of $1.21(S E=0.07)$ and $1.28(S E=0.08)$, respectively.

\section{EXPERIMENT 2}

Experiment 2 investigated the effect of a unilateral gap in noise to the left or right ear on the size of the R1 and $\mathrm{R} 2$ responses on the same and the opposite side. The issues addressed by this experiment were whether R 1 amplitude is affected by a gap, and if so, whether the effects differed on the left and right sides; whether the effect of a unilateral gap on R1 and R2 amplitude is lateralized or appears bilaterally; and finally, whether gaps to each ear differ in their effects on the size of the two components.

\section{Method}

Participants. Six volunteers (3 females) whose ages ranged from 19 to 49 years (median $=24$ years) participated. One had been tested in the previous experiment.

Apparatus and Procedure. General testing and scoring procedures were unchanged from Experiment 1 . The participants sat quietly while blink reflexes were elicited in the left and right eyes in separate trials with percutaneous stimuli delivered alone or preceded by a gap in otherwise continuous wideband noise. The noise was delivered binaurally through headphones at $55 \mathrm{~dB}$ (A) SL (measured with a Brüel and Kjær artificial ear and sound-pressure-level meter) and was present throughout the experiment. Blink reflexes were elicited by electrical stimulation of the left and right supraorbital nerves with $0.2-\mathrm{msec}$ constant-current pulses set at 1.3 times the R1-elicitation threshold current, which was determined separately for each side before the start of testing. The reflex-eliciting stimuli were presented without prestimulation to establish control reflex size (for both R1 and R2) and with a 20-msec gap in the wideband noise to either the left or the right ear whose onset preceded the ES by $100 \mathrm{msec}$. The gap was formed by gating the noise off and on over $2 \mathrm{msec}$ with a cosine function. The gap duration and the SOA were chosen to be appropriate for blink-reflex modification (Ison \& Pinckney, 1983). Six stimulus conditions were created by the factorial combination of gap side (left or right ear), ES side (left or right supraorbital nerve), and noise condition (gap or no gap). These six conditions were tested in 12 randomized blocks for a total of 72 trials. The left and right headphones were alternated from side to side for successive participants in order to counteract any difference in response characteristics of the two channels used to create and transduce the acoustic stimuli.

\section{Results and Discussion}

Figure 2 shows the mean amplitude ratios for $\mathrm{R} 1$ and the $\mathrm{R} 2$ recorded ipsilateral to the ES for gaps to the left and right ears with left- and right-sided reflex elicitation. R1 was augmented by the gap, with mean reflex amplitudes in all conditions more than double the relevant control amplitude. There were only minor differences between the conditions, and it is clear that a gap in the noise to one ear

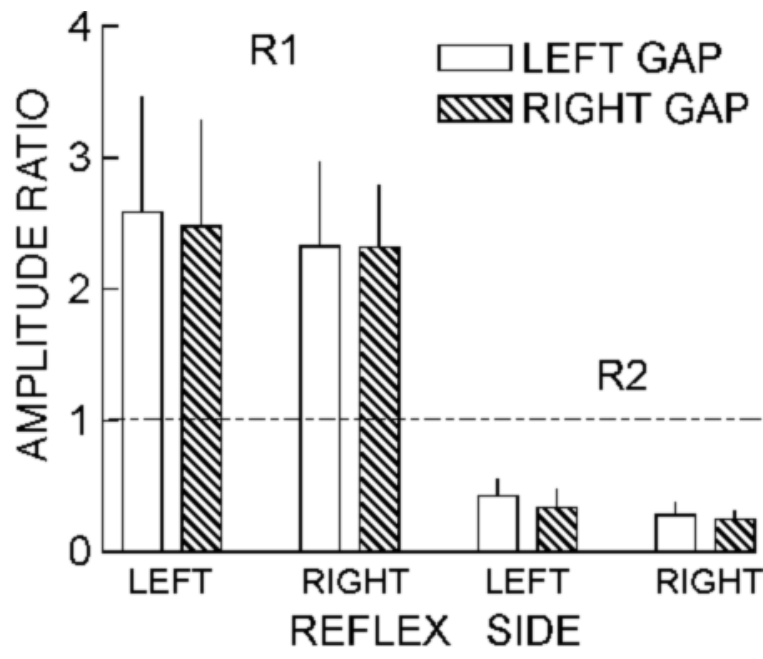

Figure 2. Mean amplitude ratios for $\mathrm{R} 1$ and the $\mathrm{R} 2$ ipsilateral to the ES on each side following gaps in noise to the left ear (open bars) and right ear (cross-hatched bars) in Experiment 2. The error bars show $\pm 1 S E$ and the broken line shows an amplitude ratio of 1.0. 
exerted a bilateral effect on R1 amplitude. The monaural gaps evoked substantial and nearly equal increases in the excitability of the R1 pathway in the brainstem both ipsilateral and contralateral to the ear in which they appeared. This result shows that the excitability of a very simple brainstem pathway is controlled by an event detected presumably in the cortex (Ison et al., 1991; Kelly et al., 1996).

$\mathrm{R} 2$ changed reciprocally to $\mathrm{R} 1$, and gaps in the noise to both left and right ears inhibited the R2 ipsilateral to the ES both on the side of the gap and contralateral to it. The results for R 2 conform to those reported by Ison and Pinckney (1983). The inhibition evoked by left- and right-ear gaps was almost the same for the R2 responses ipsilateral to an ES to the left and right supraorbital nerves. This pattern of effects of a gap in noise on the two blink components, with augmentation of $\mathrm{R} 1$ and inhibition of $\mathrm{R} 2$, is the same as that reported for a brief tone burst by Ison and Pinckney (1980). The reciprocity of the two EMG components, with augmentation of R1 and inhibition of R2, has been reported previously with warning that an ES is imminent (Ison, Sanes, Foss, \& Pinckney, 1990) and has been interpreted as showing an adaptive reduction in R2 amplitude in conditions in which an augmented R1 has initiated eyelid closure (Hammond, Thompson, Proffitt, \& Driscoll, 1996). This interpretation is consistent with the results of the previous experiment, where both R1 and R2 were augmented by closely spaced stimulus pairs; in that experiment, the stimulus configuration (with S1 below R1 threshold but above R2 threshold) would not have evoked sequential $\mathrm{R} 1$ and $\mathrm{R} 2$ responses.

The practical significance of these results is that the effects of gaps in noise to the left and right ears can be assessed with either component of the blink reflex recorded on only one side, and either ipsilateral or contralateral to the ES. Their theoretical significance is that the descending cortical control of brainstem reflex pathways, activated by a brief acoustic gap, is substantial (reflex amplitudes were roughly doubled and halved for R1 and R2 respectively) and widespread (both simple and complex pathways were affected, and the effects of unilateral gaps were present in pathways on both sides of the brainstem). Clinical observations of blink reflexes in stroke patients have shown that the excitability of both the R1 and R2 brainstem pathways is affected by damage to different cortical areas (e.g., Berardelli et al., 1983; Kimura et al., 1985). The results of this experiment complement the clinical observations to present a picture of cortical control of the brainstem pathways in which both tonic and phasic influences from widespread areas of the cerebral cortex exert bilateral control over the simple and more complex reflex pathways.

\section{EXPERIMENT 3}

Experiment 3 investigated the effect of varying the duration of lateralized gaps in noise to the left and right ears on R2 amplitude to determine whether the functions relating gap duration and reflex amplitude were asymmet- rical. Three subexperiments were run: In the first, binaural noise was presented with gaps ranging from 2 to $10 \mathrm{msec}$ in the noise to one ear or the other; in the second, monaural noise was presented with the same gap durations; and in the third, monaural noise was presented with gap durations ranging from 2 to $6 \mathrm{msec}$.

\section{Method}

Participants. Thirty-three unique participants ( 17 females) whose ages ranged from 18 to 43 years (median $=23$ years) were tested in three different subexperiments. Six were tested in two subexperiments and 1 in three to give a total of 41 separate testing sessions; participants were not tested more than once in any subexperiment.

Apparatus and Procedure. General testing and scoring procedures were unchanged from Experiments 1 and 2. The participants sat quietly while blink reflexes were elicited by brief (0.2$\mathrm{msec}$ ) constant-current pulses to the left or right supraorbital nerve at twice the R2 threshold intensity, which had been established as the minimum current required to elicit a response on three successive occasions. ESs were delivered alone to establish control reflex values, or they were preceded by a brief interruption of otherwise continuous noise to the left or right ear. The onset of the gap, which was formed in the same way as in the previous experiment, preceded the ES by $120 \mathrm{msec}$. The presentation side of the gap and gap duration were varied in each subexperiment. Intertrial intervals were selected randomly from 6 to $14 \mathrm{sec}$ with a mean of $10 \mathrm{sec}$.

In Experiment 3A, binaural $55 \mathrm{~dB}(\mathrm{~A}) \mathrm{SL}$ noise was present throughout testing. Ten participants were tested with gap durations of $2-10 \mathrm{msec}$ in $2-\mathrm{msec}$ steps. Twelve stimulus conditions were formed by the factorial combination of two gap sides (left and right ear) and six gap durations $(0-10 \mathrm{msec}$ in 2-msec steps, with the $0 \mathrm{msec}$ representing the no-gap condition). These conditions were presented in randomized blocks of 12 trials with eight replications, for a total of 96 trials. In Experiment 3B, the participants $(n=9)$ were tested under the same conditions as in Experiment 3A, with the exception that the noise was present monaurally in the left or right ear on each trial. At the end of each trial, the noise was continued in the same ear or switched to the opposite ear as required for the next trial. In both cases, the noise was present in the to-be-tested ear throughout the intertrial interval. Monaural stimulation was used to determine whether lateralizing the noise carrier as well as the gap would affect the reflex-modifying effects of the brief gaps. In Experiment $3 \mathrm{C}$, the participants $(n=22)$ were tested under the same conditions as in Experiment 3B, with the exceptions that the noise intensity was $59 \mathrm{~dB}(\mathrm{~A}) \mathrm{SL}$ and briefer gap durations (from 2 to $6 \mathrm{msec}$ in 1-msec steps) were used to examine the gap-duration functions with greater temporal resolution.

In all these experiments, the left and right headphones and the ES side were alternated for successive participants. The $\mathrm{R} 2$ responses ipsilateral to the ES were scored and analyzed with the same procedures used in the previous experiments.

\section{Results and Discussion}

Restricting the noise carrier for the gap to one ear on each trial did not affect the time course or degree of reflex inhibition, so the results of Experiments $3 \mathrm{~A}$ and $3 \mathrm{~B}$ were pooled and are shown in Figure 3. Mean reflex amplitude declined systematically with gap duration and became asymptotic with the 8-msec gap. These results confirm those of Ison and Pinckney (1983) and show additionally in a group of right-handers that gaps to the left and right ears were equally effective in inhibiting the blink reflex. The results from Experiment $3 \mathrm{C}$ with gap durations from 2 to $6 \mathrm{msec}$ in 1-msec steps are in Figure 4. They fit closely 


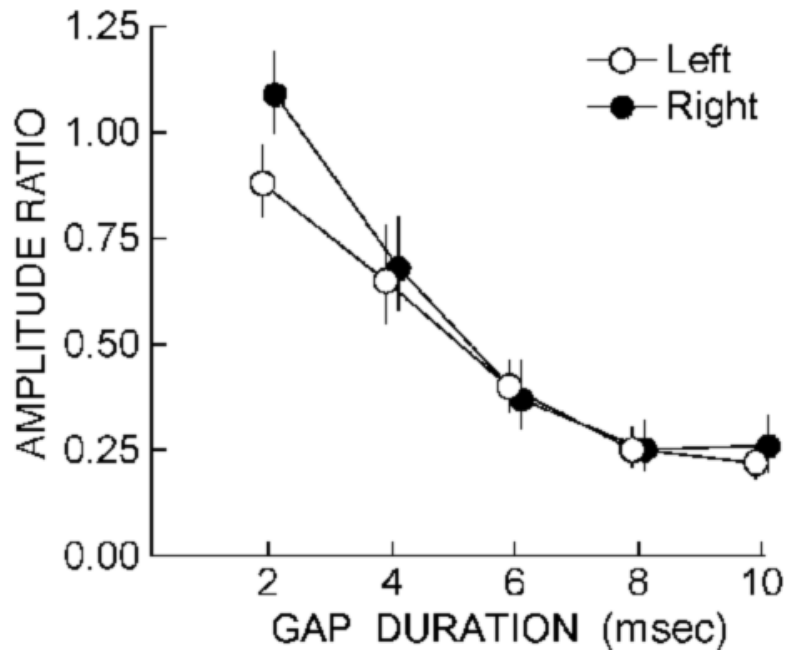

Figure 3. Mean R2 amplitude ratios following gaps in noise to the left ear (open circles) and right ear (closed circles) as a function of gap duration (in milliseconds) in Experiments $3 A$ and $3 B$. The error bars show $\pm 1 S E$. The points are slightly displaced on the abscissa for clarity.

with those obtained in Experiments $3 \mathrm{~A}$ and $3 \mathrm{~B}$ and show a systematic growth of inhibition with the gap durations tested, but no asymmetry.

Threshold durations for gaps to the left and right ears were defined for each participant as the briefest gap duration whose median blink amplitude was below the 25 th percentile of the control reflex amplitudes. Individual blinks can vary considerably in size, so a relatively stringent criterion was chosen to ensure that the threshold estimates did indicate reliable reflex inhibition. The criterion was not met at the longest duration in a few cases ( $7 \%$ of the total), with all but one of these in Experiment 3C, where the longest gap duration was $6 \mathrm{msec}$. In Experiments $3 \mathrm{~A}$ and $3 \mathrm{~B}$, in which gap durations varied with a $2-\mathrm{msec}$ step size, the mean thresholds (with $95 \%$ confidence intervals) for the left and right ears were $5.9 \mathrm{msec}(5.0-6.8 \mathrm{msec})$ and $5.6 \mathrm{msec}$ (4.7-6.4 msec), respectively; in Experiment 3C, where the step size was $1 \mathrm{msec}$, the mean thresholds (with $95 \%$ confidence intervals) for the left and right ears were $4.5 \mathrm{msec}(4.0-5.0 \mathrm{msec})$ and $4.2 \mathrm{msec}(3.6-4.9 \mathrm{msec})$, respectively. The threshold estimates from Experiments $3 \mathrm{~A}$ and $3 \mathrm{~B}$ are close to the mean value of $5.4 \mathrm{msec}$ reported by Ison and Pinckney (1983). The estimates from Experiment 3C were briefer, but still longer than the estimates of about 2-3 msec for psychophysical detection of gaps in noise (Eddins \& Green, 1995); clear evidence of inhibition in the present experiments was obtained only with gaps of $4 \mathrm{msec}$ and longer.

There was no evidence of a general asymmetry favoring gap detection by either side: The threshold gap duration was briefer for the left ear in $39 \%$ of the participants, the same for both ears in 34\% of the participants, and briefer for the right ear in $27 \%$ of the participants. The threshold estimates were relatively stable with repeated testing. Ten of 12 threshold estimates (from the two ears of the 6 participants who were tested twice) were identical or changed by one gap-duration step ( $2 \mathrm{msec}$ for Experiments $3 \mathrm{~A}$ and $3 \mathrm{~B}$ and $1 \mathrm{msec}$ for $3 \mathrm{C}$ ). There is no evidence from these data for finer temporal resolution of an acoustic stream to the right than to the left ear.

\section{GENERAL DISCUSSION}

The present experiments show that unilateral gaps in noise act bilaterally on the excitability of the brainstem pathways of the blink reflex, with a net increase in the excitability of the oligosynaptic R1 pathways and a net inhibition of the polysynaptic R2 pathways. The size of R2 was sensitive to variations in gap duration, with threshold durations for inhibition close to those derived from psychophysical measures, but there was no evidence of an asymmetry in temporal acuity from this measure. Similarly, there was no evidence that the time course of a simple brainstem process, a transient increase in excitability of reflex pathways following stimulation, differed on the left and right sides, although the magnitude of the increase was greater on the right- than on the left-hand side.

The failure to find the expected asymmetry in the effects of gaps on the blink reflex, with inhibition evoked by briefer gaps to the right than to the left ear, is in contrast to the general finding of finer temporal resolution of stimuli directed to the right than to the left sensory fields of righthanders and the specific finding of better psychophysical detection of gaps in noise to the right than to the left ear of right-handers (Brown \& Nicholls, 1997). This failure of reflex methodology to detect an asymmetry that is detected by psychophysical methods is not a result of its

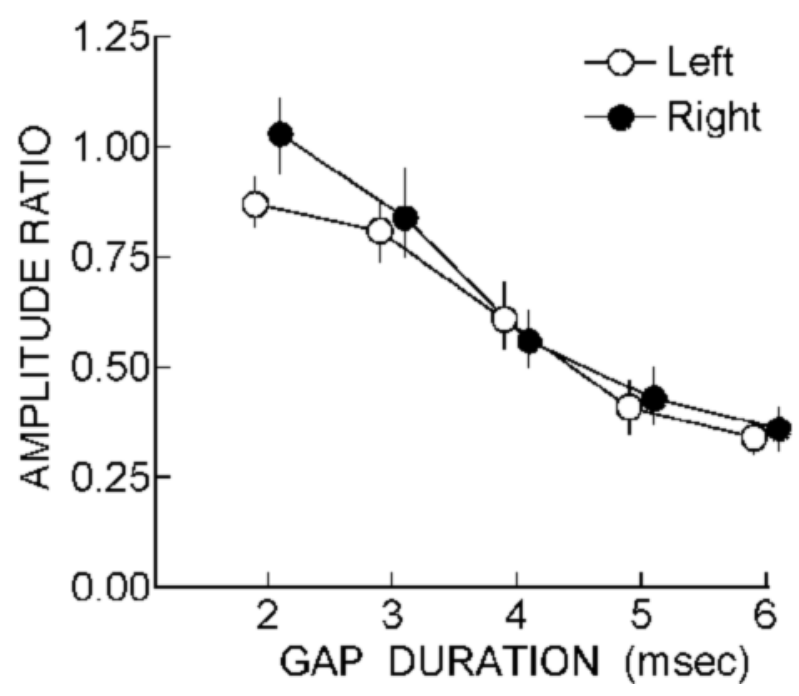

Figure 4. Mean R2 amplitude ratios following gaps in noise to the left ear (open circles) and right ear (closed circles) as a function of gap duration (in milliseconds) in Experiment 3C. The error bars show $\pm 1 S E$. The points are slightly displaced on the abscissa for clarity. 
insensitivity to temporal variation; Experiment $3 \mathrm{C}$ showed a systematic decline in relative reflex amplitude with millisecond increments in gap duration, and there was no evidence of an asymmetry with 4- and 6-msec gaps, conditions in which Brown and Nicholls found a right-ear advantage. However, despite the general similarity of threshold estimates from psychophysical and reflex procedures, they are very different methods of evaluating sensory function (Ison \& Pinckney, 1983), and indicate different endpoints of neural processing. The brain processes sensory information in different pathways for a variety of purposes, and it need not be the case that acuities characteristic of processing information in one pathway for one purpose would be the same as those characteristic of processing the same information in a different pathway for a different purpose. There may be separate auditory pathways for perception and motor control, broadly similar to the separate visual pathways for perception and action identified by Goodale and Milner (1992). The former pathway might support perception through "what" and "where" processing streams in the cortex (see, e.g., Kaas \& Hackett, 1999), and the latter might serve to modulate reflex excitability through descending projections to motor structures in the brainstem and spinal cord. A dissociation of perceptual and motor effects of acoustic input is shown by the finding that unilateral lesions of auditory cortex in man attenuated or eliminated the augmentation of a spinal reflex produced by a brief monaural tone burst to the contralesional ear despite each patient's showing a normal audiogram and with the intensity of the tone burst set relative to each patient's pure-tone threshold (Liégeois-Chauvel, Morin, Musolini, Bancaud, \& Chauvel, 1989). A similar loss of reflex modulation by tone bursts to the contralesional ear in some patients with lesions of frontal cortex led these authors to conclude that auditory modulation of the excitability of spinal reflexes is mediated by a descending system that originates in the auditory cortex and is relayed through the frontal cortex. The same system might act more widely and be responsible for auditory modulation of the excitability of the brainstem blink reflex pathway. This suggestion gets some support from studies that have implicated the medial frontal cortex in prestimulus inhibition of the acoustic startle reaction in rats (e.g., Zavitsanou, Cranney, \& Richardson, 1999).

The present results point to a symmetrical representation of the temporal structure of auditory input in each hemisphere. This conclusion is consistent with the theory advanced by Ivry and Robertson (1998), which proposes that perceptual asymmetries arise only after postsensory filtering processes that result in the low-frequency information in the input being processed in the right hemisphere and the high-frequency information being processed in the left hemisphere. In this view, hemispheric specialization is a relative bias of each hemisphere for processing low- and high-frequency regions of the input, and there is no hemispheric specialization for processing primitive stimulus features such as rapid temporal change. The present data can be interpreted in terms of this theory and the idea of separate "perception" and "action" streams of auditory processing. The higher level perceptual features that are extracted from the raw sensory input in the perception stream following high-and low-pass filtering (in the left and right hemispheres, respectively) would influence psychophysical judgments that rely on attentive processing and subjective awareness; in contrast, the descending "action" stream that controls motor excitability in lower neural levels might represent only primitive features of the unfiltered sensory input. In this way, acuities that emerge from high-level cortical processing may not be present in the modulation of sensorimotor excitability in brainstem and spinal pathways, where representation of stimulus features such as precise temporal structure might be less important.

\section{REFERENCES}

Berardelli,A., Accornero, N., Cruccu, G., Fabiano, F., Guerrisi, V., \& MANFredi, M. (1983). The orbicularis oculi response after hemispheral damage. Journal of Neurology, Neurosurgery, \& Psychiatry, 46, 837-843.

Brown, S., \& Nicholls, M. E. R. (1997). Hemispheric asymmetries for the temporal resolution of brief auditory stimuli. Perception \& Psychophysics, 59, 442-447.

Eddins, D. A., \& Green, D. M. (1995). Temporal integration and temporal resolution. In B. C. J. Moore (Ed.), Hearing (pp. 207-242). San Diego: Academic Press.

Goodale, M. A., \& Milner, A. D. (1992). Separate visual pathways for perception and action. Trends in Neurosciences, 15, 20-25.

Goode, D. J., Glenn, S., Manning, A. A., \& Middleton, J. F. (1980). Lateral asymmetry of the Hoffmann reflex: Relation to cortical laterality. Journal of Neurology, Neurosurgery, \& Psychiatry, 43, 831-835.

Hammond, G. R. (1982). Hemispheric differences in temporal resolution. Brain \& Cognition, 1, 95-118.

Hammond, G. [R.], Driscoll. P., \& Rowley, R. (1997). Temporal integration shown in the late component of the human blink reflex. Psychobiology, 25, 59-65.

Hammond, G. [R.], \& Mann, G. (1985). Bilateral symmetry of the time course of early facilitation of the Hoffmann reflex. Physiological Psychology, 13, 268-270.

Hammond, G. [R.], \& Plant, Y. (1993). Augmentation of the early component of the human blink reflex with closely spaced stimulus pairs. Psychobiology, 21, 69-76.

Hammond, G. [R.], Thompson, T., Proffitt, T., \& Driscoll, P. (1996). Functional significance of the early component of the human blink reflex. Behavioral Neuroscience, 110, 7-12.

Hoffman, H. S., \& Ison, J. R. (1980). Reflex modification in the domain of startle: I. Some empirical findings and their implications for how the nervous system processes sensory input. Psychological Review, $\mathbf{8 7}$, 175-189.

HoFFMAN, H. S., \& IsON, J. R. (1992). Reflex modification and the analysis of sensory processing in developmental and comparative research. In B. A. Campbell, H. Hayne, \& R. Richardson (Eds.), Attention and information processing in infants and adults (pp. 83-111). Hillsdale, NJ: Erlbaum.

Ison, J. R., \& HofFman, H. S. (1983). Reflex modification in the domain of startle: II. The anomalous history of a robust and ubiquitous phenomenon. Psychological Bulletin, 94, 3-17.

Ison, J. R., O'Connor, K., Bowen, G. P., \& Bocirnea, A. (1991). Temporal resolution of gaps in noise by the rat is lost with functional decortication. Behavioral Neuroscience, 105, 33-40.

Ison, J. R, \& PincKNeY, L. [A.] (1980). Reflex modulation in humans by monaural and binaural auditory stimulation. Bulletin of the Psychonomic Society, 15, 285-287.

Ison, J. R, \& Pinckney, L. A. (1983). Reflex inhibitionin humans: Sensitivity to brief silent periods in white noise. Perception \& Psychophysics, 34, 84-88.

Ison, J. R, Sanes, J. N., Foss, J. A., \& Pinckney, L. A. (1990). Facilita- 
tion and inhibition of the human startle blink reflexes by stimulus anticipation. Behavioral Neuroscience, 104, 418-429.

IVRY, R. B., \& RoberTSON, L. C. (1998). The two sides of perception. Cambridge, MA: MIT Press.

KAAS, J. H., \& HACKeTt, T. A. (1999). "What" and "where" processing in auditory cortex. Nature Neuroscience, 2, 1045-1047.

Kelly, J. B., Rooney, B. J., \& Phillips, D. P. (1996). Effects of bilateral auditory cortical lesions on gap-detection thresholds in the ferret (Mustela putorius). Behavioral Neuroscience, 110, 542-550.

Kimura, J., Wilkinson, J. T., Damasio, H., Adams, H. R., Shivapour, E. \& Yamada, T. (1985). Blink reflex in patients with hemispheric cerebrovascular accident (CVA). Journal of the Neurological Sciences, $\mathbf{6 7}$, $15-28$.

Liégeois-Chauvel, C., Morin, C., Musolini, A., Bancaud, J., \& Chauvel, P. (1989). Evidence for a contribution of the auditory cortex to audiospinal facilitation in man. Brain, 112, 375-391.

Nicholls, M. E. R. (1996). Temporal processing asymmetries between the cerebral hemispheres: Evidence and implications. Laterality, 1, 97-137.
Tallal, P., Galaburda, A. M., Llinas, R R, \& von Euler, C. (Eds.) (1993). Temporal information processing in the nervous system: Special reference to dyslexia and dysphasia (Annals of the New York Academy of Sciences, Vol. 682). New York: New York Academy of Sciences. Trehub, S. E., Schneider, B. A., \& Henderson, J. L. (1995). Gap detection in infants, children, and adults. Journal of the Acoustical Society of America, 98, 2532-2541.

Trevarthen, C. (1996). Lateral asymmetries in infancy: Implications for the development of the hemispheres. Neuroscience \& Biobehavioral Reviews, 20, 571-586.

Zavitsanou, K., Cranney, J., \& Richardson, R. (1999). Dopamine antagonists in the orbital prefrontal cortex reduce prepulse inhibition of the acoustic startle reflex in the rat. Pharmacology, Biochemistry \& Behavior, 63, 55-61.

(Manuscript received December 13, 2000; revision accepted for publication July 17,2001 .) 